\title{
Reductive Conjugate Addition nitro-Mannich Route for the Stereoselective Synthesis of 1,2,3,4-Tetrahydroquinoxalines
}

James C. Anderson, ${ }^{* a}$ Ian B. Campbell, ${ }^{b}$ Sebastien Campos, ${ }^{b}$ lain H. Reid, ${ }^{b}$ Christopher D. Rundell, a Jonathan Shannon ${ }^{\mathrm{a}}$ and Graham J. Tizzard $\$ c$

aDepartment of Chemistry, University College London, 20 Gordon Street, London, WC1H 0AJ, UK. E-mail: j.c.anderson@ucl.ac.uk

${ }^{\mathrm{b}}$ GlaxoSmithKline, Medicines Research Centre, Stevenage, SG1 2NY, UK.

'National Crystallography Service, School of Chemistry, university of Southampton, Southampton SO17 1BJ, UK.

${ }^{\ddagger}$ Corresponding author for crystallographic results. E-Mail: gjt1@soton.ac.uk

Electronic supplementary information (ESI) available: General experimental details, copies of ${ }^{1} \mathrm{H}$ and ${ }^{13} \mathrm{C}$ NMR spectra. See DOI: 10.1039/x0xx00000x

Abstract. A concise, high yielding and structurally divergent synthesis of complex 1,2,3,4-tetrahydroquinoxalines with excellent diastereoselectivity is described. A wide array of nitroalkenes and imines derived from commercially available aromatic aldehydes and 2-chloroanalines were subjected to a key reductive conjugate addition nitro-Mannich reaction to give diastereomerically pure $\beta$-nitro amines. Sequential reduction of the nitro function followed by Pd-catalyzed intramolecular $\mathrm{N}$-arylation of the resultant primary amine onto the 2-chloroanailine gives highly substituted 1,2,3,4tetrahydroquinoxalines. Non basic imines were found to participate better in the nitroMannich reaction if the stronger acid methanesulfonic acid was used to promote the reaction. The 3 step reaction sequence should be useful for the array synthesis of drug like scaffolds.

\section{Introduction.}

The 1,2,3,4-tetrahydroquinoxaline scaffold has become increasingly important in medical chemistry. ${ }^{1}$ Several compounds containing the 1,2,3,4-tetrahydroquinoxaline core have demonstrated biological activities against a range of targets including as 
potent cholesteryl ester transfer protein inhibitors, ${ }^{1 \mathrm{a}}$ vasopressin V2 receptor antagonists, ${ }^{1 \mathrm{~b}}$ and prostaglandin D2 receptor antagonists, ${ }^{1 \mathrm{c}}$ (Figure 1). Despite the marked interest in 1,2,3,4-tetrahydroquinoxalines there are only a limited number of synthetic methods available. ${ }^{2}$ The most common approach for the synthesis of tetrahydroquinoxalines is the hydrogenation of quinoxalines.

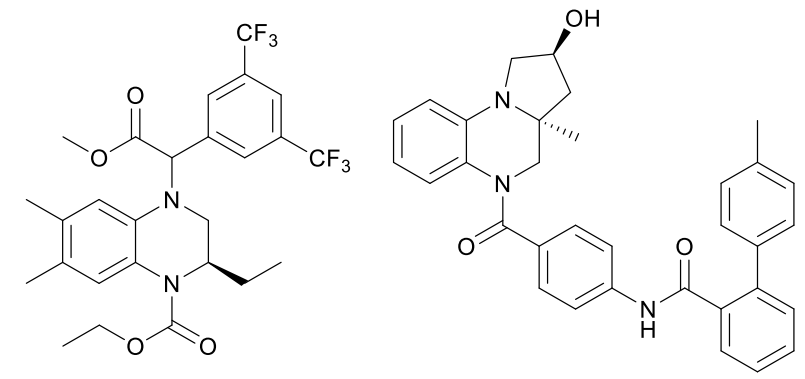

Figure 1: Biologically active 1,2,3,4-tetrahydroquinoxalines.

We have investigated the use of the nitro-Mannich reaction ${ }^{3}$ in the synthesis of stereochemically defined heterocyclic ring systems. ${ }^{4}$ In order to access more complex heterocycles we have expanded the structures of possible nitroalkane reaction partners through the in situ conjugate addition of nucleophiles to nitroalkenes, with trapping of the subsequent nitronate species with an imine partner. ${ }^{5}$ We have shown that this procedure can be used to generate more complex stereodefined $\beta$-nitroamines that can be used in heterocycle synthesis. ${ }^{6}$ As part of an investigation into using the nitro-Mannich reaction in diversity-oriented array synthesis, we were interested in the synthesis of arrays of stereochemically diverse fused heterocyclic ring systems. ${ }^{7}$ We have shown that the reductive nitro-Mannich reaction with arylbromide nitrostyrenes 1 can deliver stereodefined functionalised diamine building blocks 2 that are precursors to either 3-aminotetrahydroquinolines $\mathbf{3}$ or 2-aminomethylene indolines $\mathbf{4}$ via $\mathrm{N}$-arylation (Scheme 1). ${ }^{7 \text { a }}$ More recently we demonstrated that placing the $\mathrm{N}$-arylation cyclisation handle on the imine precursor 5, derived from an ortho-bromine substituted aromatic aldehyde gave 1,2-diamine containing indolines 6 (Scheme 1)..$^{7 \mathrm{~b}}$ In this paper we detail an alternative combination leading to the stereoselective synthesis of an array of 1,2,3,4tetrahydroquinoxalines 7 (Scheme 2). Disconnection of 7 using a key $\mathrm{N}$-arylation leads to diamine 8 which can be derived from reduction of $\beta$-nitroamine $\mathbf{9}$. This can be accessed from a stereoselective reductive nitro-Mannich reaction ${ }^{5 a, b}$ between 
nitroalkene $\mathbf{1 0}$ and imine $\mathbf{1 1}$. In this combination the $\mathrm{N}$-arylation cyclisation handle is part of the aryl amine of the imine 11. The nitroalkenes can be prepared from a Henry reaction and the imine from an ortho-halo amine and aldehyde. An important feature of the strategy is the ease in which structural diversity can be introduced into the products. This arises from the large selection of aromatic aldehydes that can be used to form nitroalkene 10, as well as the ortho-halo amines and aldehydes used to form imine 11. This provides the opportunity for the synthesis of an array of novel fused heterocyclic structures which we exemplify here.

\section{Scheme 1. Previous work}

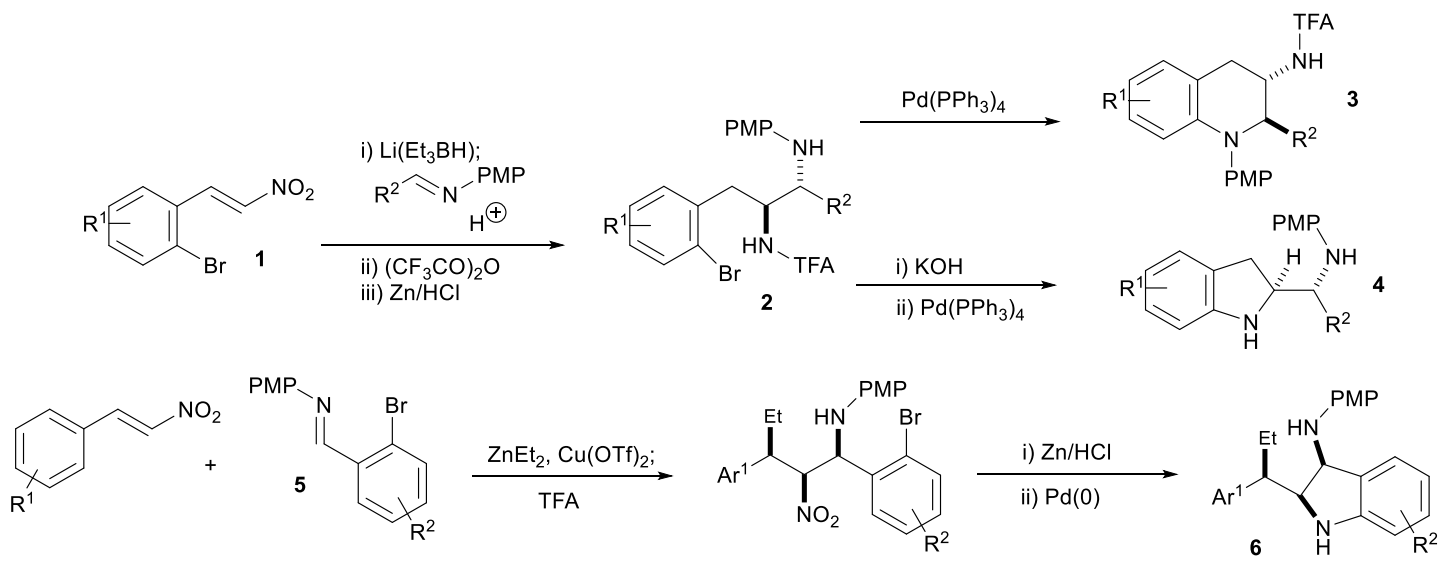

Scheme 2. This work

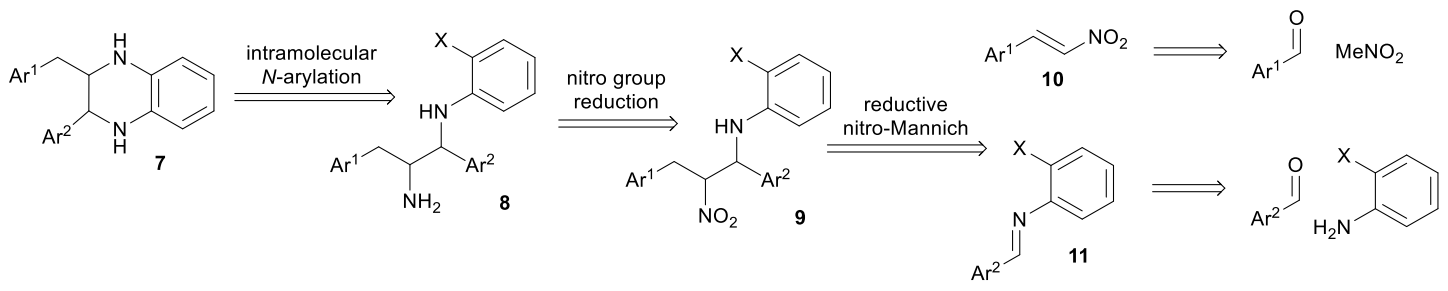

\section{Results and discussion}

The first step in the synthetic strategy was the one-pot reductive nitro-Mannich reaction between nitrostyrenes $\mathbf{1 0}$ and imines $\mathbf{1 1}$ which was performed with lithium triethylborohydride according to our previously published procedure, ${ }^{5 b}$ furnishing $\beta$ nitroamines 9 in excellent conversion and diastereoselectivity (Scheme 3, Table 1).

\section{Scheme 3. Stereoselective reductive nitro-Mannich reaction}




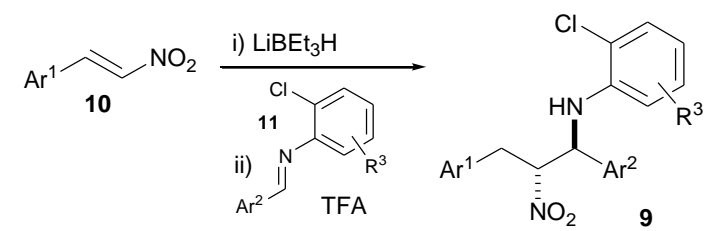

Table 1. Scope of $\beta$-nitroamine ${ }^{a}$

\begin{tabular}{|c|c|c|c|c|c|c|}
\hline Product & $A r^{1}$ & $\mathrm{Ar}^{2}$ & $\mathrm{R}^{3}$ & Conversion (\%) & $9 d r$ crude $^{b}$ & Yield $9(\%)^{c}$ \\
\hline $9 a$ & $\mathrm{Ph}$ & $\mathrm{Ph}$ & $H^{d}$ & $>95$ & $90: 10$ & 68 \\
\hline $9 b$ & $\mathrm{Ph}$ & $\mathrm{Ph}$ & $\mathrm{H}$ & $>95$ & $95: 5$ & 72 \\
\hline 9c & 4-MePh & $\mathrm{Ph}$ & $\mathrm{H}$ & $>95$ & $90: 10$ & 75 \\
\hline $9 d$ & 2-MePh & $\mathrm{Ph}$ & $\mathrm{H}$ & $>95$ & $90: 10$ & 70 \\
\hline $9 e$ & 4-MeO-Ph & $\mathrm{Ph}$ & $\mathrm{H}$ & $>95$ & $90: 10$ & 74 \\
\hline $9 f$ & 2-MeO-Ph & $\mathrm{Ph}$ & $\mathrm{H}$ & $>95$ & $85: 15$ & 71 \\
\hline $9 g$ & 3-py & $\mathrm{Ph}$ & $\mathrm{H}$ & $>95$ & $>95: 5$ & 67 \\
\hline $9 \mathrm{~h}$ & $\mathrm{Ph}$ & $\mathrm{Ph}$ & $4-{ }^{t} \mathrm{Bu}$ & $>95$ & $90: 10$ & 79 \\
\hline $9 i$ & $\mathrm{Ph}$ & $\mathrm{Ph}$ & $4-F$ & $>95$ & $90: 10$ & 65 \\
\hline $9 j$ & $\mathrm{Ph}$ & $\mathrm{Ph}$ & 4-OMe & $>95$ & $85: 15$ & 72 \\
\hline $9 k$ & $\mathrm{Ph}$ & $\mathrm{Ph}$ & 6-Me & 45 & $55: 45$ & $-^{\mathrm{e}}$ \\
\hline 9l & $\mathrm{Ph}$ & $\mathrm{Ph}$ & $5-\mathrm{CF}_{3}$ & 50 & $90: 10$ & $-{ }^{e}$ \\
\hline $9 m$ & $\mathrm{Ph}$ & $\mathrm{Ph}$ & $3-p y^{f}$ & $>95$ & $90: 10$ & 61 \\
\hline $9 n$ & $\mathrm{Ph}$ & 3-MeO-Ph & $\mathrm{H}$ & $>95$ & $95: 5$ & 72 \\
\hline 90 & $\mathrm{Ph}$ & $3-\mathrm{CF}_{3}-\mathrm{Ph}$ & $\mathrm{H}$ & $>95$ & $90: 10$ & $63^{g}$ \\
\hline $9 p$ & $\mathrm{Ph}$ & 2-Me-Ph & $\mathrm{H}$ & $>95$ & $80: 20$ & $-\mathrm{g}$ \\
\hline $9 q$ & $\mathrm{Ph}$ & 3-furyl & $\mathrm{H}$ & $>95$ & $75: 25$ & 70 \\
\hline $9 r$ & $\mathrm{Ph}$ & 3-py & $\mathrm{H}$ & $40^{\mathrm{h}}$ & $90: 10$ & $-{ }^{e}$ \\
\hline $9 s$ & $\mathrm{Ph}$ & & $\mathrm{H}$ & $>95$ & $80: 20$ & $-i$ \\
\hline $9 t$ & $\mathrm{Ph}$ & & $\mathrm{H}$ & $>95$ & $80: 20$ & $-^{i}$ \\
\hline
\end{tabular}

${ }^{a}$ Nitro-Mannich: 10 (3 mmol), superhydride ${ }^{\circledR}$ (1.05 equiv. $1 \mathrm{M}$ in THF), $\mathrm{CH}_{2} \mathrm{Cl}_{2}, \mathrm{rt}, 1 \mathrm{~h} ; 11$ (1.5 equiv.), TFA (2.6 equiv.), $\mathrm{CH}_{2} \mathrm{Cl}_{2},-78^{\circ} \mathrm{C}, 1 \mathrm{~h}$ then rt $1 \mathrm{~h}$. biastereoselectivities were calculated by comparison of the ${ }^{1} \mathrm{H}$ NMR signals for the $\mathrm{CH}_{2} \mathrm{CHNO}_{2}$ protons $(\sim \delta$ 3.1-3.6 ppm) of the crude reaction mixture. 
Isolated yield of pure anti-diastereoisomer. ${ }^{d} 2-\mathrm{Br}$ instead of $2-\mathrm{Cl}$. ${ }^{e}$ Reaction not purified. ${ }^{f}$ Imine derived from 3-amino-2-chloropyridine. ${ }^{g}$ Diastereoisomers inseparable. ${ }^{h}$ TFA (2.6 equiv.). 'Decomposed on silica chromatography.

The $\beta$-nitroamines 9 were tentatively assigned the anti-stereochemistry based upon the $1 \mathrm{H}$ NMR chemical shifts $(\delta)$ and coupling constants $(\mathrm{J})$ recorded for the protons adjacent to the two nitrogen atoms. In our nitro-Mannich system the vast majority of products have given anti-selectivity, for which we have forwarded a transition state model. ${ }^{8,9}$ The assignment of these $\beta$-nitroamines 9 was corroborated by single crystal X-ray analysis of a derivative of $\mathbf{9 b}$ (see ESI). Due to the relative instability of $\beta$-nitroamines these products are usually isolated as their trifluoroacetamides, by treatment of the crude reaction mixtures with TFAA in the presence of pyridine. Unusually these particular $\beta$-nitroamines (9) were relatively stable to silica chromatography and could be isolated as single diastereoisomers and used directly in the next steps of the reaction sequence towards the 1,2,3,4tetrahydroquinoxalines 7 .

The Nitrostryene component $\mathbf{1 0}$ had the broadest compatibility $\left(\operatorname{Ar}^{1}\right)$, with electron rich, electron deficient and a pyridyl group all showing good conversion and diastereoselectivity (9a-g). A 2-halo substituent was necessary in the aniline group to enable an $\mathrm{N}$-arylation cyclisation. Although the ortho-bromoanailine derived imine worked well in this nitro-Mannich reaction (9a), there was a wider diversity of anilines available that possessed the ortho-chloro substituent $\left(R^{3}\right)$. The orthochloroanilines were found to be able to tolerate, sterically bulky, electron rich and electron deficient substituents as well as a nitrogen containing heterocycle (Entries $\mathbf{9 h}-\mathbf{j}, \mathbf{9 m}$ ) in this reaction. The reaction struggled with conversion when there was steric congestion close to the imine (9k) and gave virtually no diastereoselectivity. The deactivating $5-\mathrm{CF}_{3}$ substituent reduced conversion to $\sim 50 \%(9 \mathrm{I})$, but gave good diastereoselectivity. The most sensitive component to change was the aromatic aldehyde substituent of the imine $\mathbf{1 1}\left(\mathrm{Ar}^{2}\right)$, which tolerated some electron donating (9n), electron withdrawing (9o) substituents, but gave either a low conversion or diastereoselectivity with 2-substituents (9p) and heterocyclic rings (9q-t). The diastereoisomers of product 90 derived from 3-trifluorobenzaldehyde could not be 
separated and were isolated as a 90:10 mixture. Similarly the diastereoisomers of product $9 p$ derived from 2-methylbenzaldehyde could not be separated and due to the poorer diastereoselectivity (80:20) was not investigated further. The products derived from the $\mathrm{N}$-tosylpiperidine imines $(\mathbf{9 s}, \mathbf{t})$ were unstable towards chromatography.

It was notable that lower conversion was observed when the substituent of the imine could affect the $\mathrm{p} K_{\mathrm{a}} \mathrm{H}$ of the imine (for example $9 \mathrm{l}$ and $9 \mathrm{r}$ ). Previous nitroMannich reactions from our work have demonstrated that the imine partner needs to be protonated for these particular nitro-Mannich reactions, that is with an alkyl or aryl substituent on the nitrogen of the imine, to proceed. ${ }^{8,7 b}$ The $\mathrm{p} K_{\mathrm{a}} \mathrm{H}\left(\mathrm{H}_{2} \mathrm{O}\right)$ of several imines were measured in order to probe this phenomenon more accurately (See ESI). The PMP protected imine 12 works well in nitro Mannich reactions ${ }^{7 \mathrm{~b}}$ and has a $\mathrm{p} K_{\mathrm{a}} \mathrm{H}$ 5.4. The standard 2-chloroaniline derived imine 11b has $\mathrm{p} K_{\mathrm{a}} \mathrm{H} 2.8$ and appears to function well in this reaction (Table 1, 9b). The 2-chloro-5trifluoromethylaniline derived imine $11 \mathrm{l}$ has $\mathrm{p} K_{\mathrm{a}} \mathrm{H}<2.0$ and only gave $50 \%$ conversion under our standard conditions with TFA (Table 1, 9l). This is in contrast to having the trifluoromethyl group on the aldehyde portion of the imine. Imine $\mathbf{1 1 0}$ has $\mathrm{p} K_{\mathrm{a}} \mathrm{H} 2.7$ and functions well in these reactions (9o). Imine $\mathbf{1 1 r}$ derived from pyridine-3carboxaldehyde has $\mathrm{p} K_{\mathrm{a}} \mathrm{H} 2.9$, but due to the pyridine group. Presumably under the acidic conditions the pyridine is protonated and this has an adverse effect on the basicity of the imine lone pair, significantly reducing conversion to $40 \%$ with TFA (Table 1, 9r). In an attempt to improve conversion, other acids were screened in the reductive nitro-Mannich reaction (Scheme 3 and Table 2), with imine 11r. It was found that the stronger acid $\mathrm{MeSO}_{3} \mathrm{H}$ gave the best result for imine $11 \mathrm{r}$ with a $64 \%$ yield of pure anti- $\beta$-nitroamine $9 r$ isolated. In this case at least two equivalents of $\mathrm{MeSO}_{3} \mathrm{H}$ were required for complete conversion. This suggested that the pyridine nitrogen is protonated first, which lowers the $\mathrm{p} K_{\mathrm{a}} \mathrm{H}$ of the imine nitrogen, and therefore a stronger acid is required to protonate the imine nitrogen. Imine $\mathbf{1 1 l}$ with $\mathrm{MeSO}_{3} \mathrm{H}$ gave a much improved conversion (>95\%) compared to only $50 \%$ with TFA (Table 1), resulting in a $61 \%$ isolated yield of $9 \mathrm{l}$, but as a $90: 10$ mixture of diastereoisomers. The diastereoselectivity remained unaltered, as it also did for the imine 11q derived from furan-3-carboxaldehyde (cf Table 1, 9q). 
Figure 2. $\mathrm{pK}_{\mathrm{a}}\left(\mathrm{H}_{2} \mathrm{O}\right)$ of selected imines<smiles>COc1ccc(N=Cc2ccccc2)cc1</smiles>

Table 2. Effect of acid on reductive nitro-Mannich reaction ${ }^{a}$

\begin{tabular}{|c|c|c|c|c|}
\hline Imine & Acid & Conversion (\%) & $9 d r$ crude $^{b}$ & Yield 9 (\%) \\
\hline $11 r$ & TFA & 40 & $90: 10$ & $-{ }^{c}$ \\
\hline $11 r$ & $\mathrm{AcOH}$ & 0 & - & - \\
\hline $11 r$ & Citric & 0 & - & - \\
\hline $11 r$ & $\mathrm{MeSO}_{3} \mathrm{H}$ & $>95$ & $95: 5$ & $64^{d}$ \\
\hline $11 r$ & $\mathrm{CF}_{3} \mathrm{SO}_{3} \mathrm{H}$ & $>95$ & $90: 10$ & $50^{d}$ \\
\hline $11 r$ & $\mathrm{HCO}_{2} \mathrm{H}$ & 35 & $85: 15$ & $-c$ \\
\hline $11 r$ & Maleic & 30 & $85: 15$ & $-^{c}$ \\
\hline $11 r$ & $p-\mathrm{MePhSO}_{3} \mathrm{H}$ & 0 & - & - \\
\hline 111 & $\mathrm{MeSO}_{3} \mathrm{H}$ & $>95^{e}$ & $90: 10$ & $61^{f}$ \\
\hline $11 q$ & $\mathrm{MeSO}_{3} \mathrm{H}$ & $>95^{e}$ & $75: 25$ & $-^{c}$ \\
\hline
\end{tabular}

${ }^{a}$ Nitro-Mannich: 10 (3 mmol), superhydride ${ }^{\circledR}$ (1.05 equiv. $1 \mathrm{M}$ in $\mathrm{THF}$ ), $\mathrm{CH}_{2} \mathrm{Cl}_{2}, \mathrm{rt}, 1 \mathrm{~h} ; 11$ (1.5 equiv.), Acid (2.6 equiv.), $\mathrm{CH}_{2} \mathrm{Cl}_{2},-78{ }^{\circ} \mathrm{C}, 1 \mathrm{~h}$ then $\mathrm{rt} 1 \mathrm{~h}$. ${ }^{\mathrm{b}}$ Diastereoselectivities were calculated by comparison of the ${ }^{1} \mathrm{H}$ NMR signals for the $\mathrm{CH}_{2} \mathrm{CHNO}_{2}$ protons ( $\sim$ 3.1-3.6 ppm) of the crude reaction mixture. ${ }^{c}$ Reaction not purified. ${ }^{d}$ Isolated yield of pure anti-diastereoisomer. ${ }^{e} \mathrm{MeSO}_{3} \mathrm{H}$ (1.6 equiv.). ${ }^{f}$ Isolated as a 90:10 mixture of diastereoisomers.

The desired intramolecular $N$-arylation reaction required the nitro group to be reduced with zinc dust and hydrochloric acid in ethanol. The crude anti-1,2-diamine $\mathbf{8}$ was treated directly with X-Phos and palladium acetate to provide diastereomerically pure 1,2,3,4-tetrahydroquinoxalines 7 in excellent yield (Scheme 4, Table 3). The X-phos ligand was required instead of the standard $N$-arylation conditions we have used previously $^{7 \mathrm{a}, \mathrm{b}}$ because of the lower reactivity of the chloride substituent in $\mathrm{N}$-arylation reactions. ${ }^{11}$

\section{Scheme 4. Synthesis of 1,2,3,4-tetrahydroquinoxalines 7}




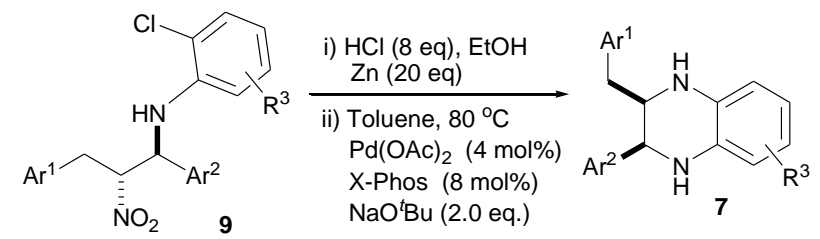

Table 3. Diversity of 1,2,3,4-tetrahydroquinoxalines $7^{a}$

\begin{tabular}{lllll}
\hline Product & $\mathrm{Ar}^{1}$ & $\mathrm{Ar}^{2}$ & $\mathrm{R}^{3}$ & $\begin{array}{l}\text { Yield } \\
(\%)^{b}\end{array}$ \\
\hline 7b & $\mathrm{Ph}$ & $\mathrm{Ph}$ & $\mathrm{H}$ & 80 \\
7c & 4-Me-Ph & $\mathrm{Ph}$ & $\mathrm{H}$ & 74 \\
7d & 2-Me-Ph & $\mathrm{Ph}$ & $\mathrm{H}$ & 70 \\
7e & 4-MeO-Ph & $\mathrm{Ph}$ & $\mathrm{H}$ & 77 \\
7f & 2-MeO-Ph & $\mathrm{Ph}$ & $\mathrm{H}$ & 68 \\
7h & $\mathrm{Ph}$ & $\mathrm{Ph}$ & $4-{ }^{-} \mathrm{Bu}$ & 76 \\
7i & $\mathrm{Ph}$ & $\mathrm{Ph}$ & $4-\mathrm{F}$ & 63 \\
7j & $\mathrm{Ph}$ & $\mathrm{Ph}$ & $4-\mathrm{OMe}$ & 73 \\
7l & $\mathrm{Ph}$ & $\mathrm{Ph}$ & $5-\mathrm{CF}_{3}$ & $73^{c}$ \\
7m & $\mathrm{Ph}$ & $\mathrm{Ph}$ & $3-\mathrm{py}^{c}$ & 61 \\
7n & $\mathrm{Ph}$ & $3-\mathrm{MeO}-\mathrm{Ph}$ & $\mathrm{H}$ & 69 \\
70 & $\mathrm{Ph}$ & $3-\mathrm{CF}_{3}-\mathrm{Ph}$ & $\mathrm{H}$ & 71 \\
7r & $\mathrm{Ph}$ & $3-\mathrm{py}$ & $\mathrm{H}$ & 69
\end{tabular}

${ }^{a}$ Reduction: 9 ( $\left.3 \mathrm{mmol}\right), \mathrm{Zn}$ (8 equiv) $\mathrm{HCL}(6 \mathrm{M}, 20$ equiv) in $\mathrm{EtOH}(50 \mathrm{ml})$ at rt for $1 \mathrm{~h}$. Cyclisation: Crude diamine 8 (3 mmol 1.0 eq.), $\mathrm{NaO}^{t} \mathrm{Bu}$ (2 eq.), X-Phos $\left(0.040 \mathrm{mmol}, 8 \mathrm{~mol} \%\right.$ ) and $\mathrm{Pd}(\mathrm{OAc})_{2}(4 \mathrm{~mol}$ $\%)$ in PhMe $(2.5 \mathrm{~mL})$ at $90{ }^{\circ} \mathrm{C}$ for 3-12 hrs until complete conversion. ${ }^{b}$ Isolated yield of diastereomerically pure product over two steps. 'Isolated as a 90:10 mixture of diastereoisomers

Notably $\beta$-nitroamines $\mathbf{9 g}$ and $\mathbf{9 q}$ decomposed during the palladium catalysed $N$ arylation cyclisation step. Also 1,2,3,4-tetrahydroquinoxaline $\mathbf{7 l}$ was isolated as an inseparable 90:10 mixture. Presumably some minor epimerisation occurred during the reduction step.

Extension of this methodology by using a carbon nucleophile in the initial conjugate addition nitro-Mannich reaction was investigated using $\mathrm{Et}_{2} \mathrm{Zn} / \mathrm{Cu}(\mathrm{OTf})_{2}$ cat. $^{5 \mathrm{a}}$ and Grignard reagents. Variable diastereoselection, poor diastereoselctivity (60:40 to 
$80: 20)$ and yields below $40 \%$ were observed. These results suggest that the 2 chloroaniline imine substituent is not suited to that particular reaction.

\section{Conclusions}

We have devised a synthetic strategy for the preparation of highly substituted 1,2,3,4tetrahydroquinoxalines which uses the reductive conjugate addition nitro-Mannich reaction as a key stereochemical determining reaction in excellent yields and distereoselectivities. Subsequent nitro reduction followed by Pd-catalyzed intramolecular $N$-arylation completes the reactions sequence. Non basic imines were found to participate better in the nitro-Mannich reaction if the stronger acid methanesulfonic acid was used to promote the reaction instead of trifluoroacetic acid. This may enable other more difficult nitro-Mannich reactions to proceed. The reaction sequence is ideally suited to array synthesis as it allows the use of a wide range of aromatic aldehydes in the nitroalkene and imine reaction partners, with 2-chloro anilines.

\section{Experimental}

General Methods - please see ESI

General Procedure for the Synthesis of anti- $\beta$-Nitroamines 9 (Table 1)

A solution of superhydride ${ }^{\circledR}(3.15 \mathrm{mmol}, 1 \mathrm{M}$ in THF) was added to a solution of nitroalkene 10 (3 mmol) in $\mathrm{CH}_{2} \mathrm{Cl}_{2}(15 \mathrm{~mL})$ under $\mathrm{N}_{2}$ at $\mathrm{rt}$ and stirred for $1 \mathrm{~h}$. The white suspension was then cooled to $-78{ }^{\circ} \mathrm{C}$, a solution of imine $11(4.5 \mathrm{mmol})$ in $\mathrm{CH}_{2} \mathrm{Cl}_{2}(5 \mathrm{~mL})$ was added and stirred for $5 \mathrm{~min}$. Then a solution of TFA $(4.8 \mathrm{mmol})$ in $\mathrm{CH}_{2} \mathrm{Cl}_{2}(1 \mathrm{~mL})$ was then added dropwise, and the reaction stirred at $-78^{\circ} \mathrm{C}$ for $1 \mathrm{~h}$, removed from the cold bath, stirred for a further $1 \mathrm{~h}$ at RT, and then quenched with saturated aq. $\mathrm{NaHCO}_{3}(15 \mathrm{~mL})$. The layers were separated, the aqueous phase further extracted with $\mathrm{CH}_{2} \mathrm{Cl}_{2}(2 \times 20 \mathrm{~mL})$, the combined organics washed with brine $(10 \mathrm{~mL})$, dried $\left(\mathrm{MgSO}_{4}\right)$, and the solvent removed in vacuo to give crude $\beta$ nitroamine. Diastereoselectivities were calculated by comparison of the $1 \mathrm{H} N \mathrm{NM}$ signals for the $\mathrm{CH}_{2} \mathrm{CHNO}_{2}$ protons ( $\delta$ 3.1-3.6 ppm). The crude $\beta$-nitroamine was then purified by column chromatography to yield diastereomerically pure, anti- $\beta$ nitroamine 9 .

$\left(2 R^{*}, 3 S^{*}\right)$-2-Bromo-N-(2-nitro-1,3-diphenylpropyl)aniline (9a) 
Colourless oil; ${ }^{1} \mathrm{H}$ NMR (600 MHz; $\left.\mathrm{CDCl}_{3}\right) \delta 3.21\left(1 \mathrm{H}, \mathrm{dd}, J=14.9,3.3, \mathrm{CH}_{2 \mathrm{a}}\right), 3.46(1 \mathrm{H}$, $\left.\mathrm{dd}, J=14.9,10.5, \mathrm{CH}_{2 \mathrm{~b}}\right), 4.78(1 \mathrm{H}, \mathrm{m}, \mathrm{CHNH}), 5.03\left(1 \mathrm{H}, \mathrm{ddd}, J=10.3,5.2,3.5, \mathrm{CHNO}_{2}\right.$ ), $5.32(1 \mathrm{H}, \mathrm{d}, J=6.9, \mathrm{NH}), 6.42(1 \mathrm{H}$, app. d, $J=8.2, \operatorname{ArH}), 6.60(1 \mathrm{H}$, app. t, $J=7.6, \operatorname{ArH})$, $7.02(1 \mathrm{H}$, app. d, J =7.8, ArH), 7.11-7.14 (2H, m, ArH), 7.22-7.26 (1H, m, ArH), 7.27$7.30(2 \mathrm{H}, \mathrm{m}, \mathrm{ArH}), 7.32-7.41(5 \mathrm{H}, \mathrm{m}, \operatorname{ArH}), 7.43-7.46(1 \mathrm{H}, \mathrm{m}, \mathrm{ArH}) ;{ }^{13} \mathrm{C} N M R(151 \mathrm{MHz}$; $\left.\mathrm{CDCl}_{3}\right) \delta 34.9,60.5,93.4,110.9,112.9,119.5,127.0,127.6,128.5,128.9,129.0$, 129.1, 129.4, 132.7, 135.4, 136.7, 142.8; IR (neat) 3392, 3030, 2916, 1595, $1550 \mathrm{~cm}^{-1}$; Mass Spec (ES, M + H) Theoretical: 410.0624, Measured: 410.0634.

\section{$\left(2 R^{*}, 3 S^{*}\right)$-2-chloro-N-(2-nitro-1,3-diphenylpropyl)aniline (9b)}

White solid, mp 118-120 ${ }^{\circ} \mathrm{C} ;{ }^{1} \mathrm{H}$ NMR (400 MHz; $\left.\mathrm{CDCl}_{3}\right) \delta 3.22(1 \mathrm{H}, \mathrm{dd}, \mathrm{J}=14.8,3.5)$, $3.46(1 \mathrm{H}, \mathrm{dd}, J=14.8,10.3), 5.05$ (1H, dd, J=7.0, 5.5), 5.13 (1H, ddd, J=10.0, 5.5, 3.5), $5.28(1 \mathrm{H}, \mathrm{d}, J=7.0), 6.46(1 \mathrm{H}, \mathrm{dd}, J=8.2,1.1), 6.66(1 \mathrm{H}, \mathrm{td}, J=7.7,1.5), 6.97-7.03(1 \mathrm{H}$, m), $7.12(2 \mathrm{H}, \mathrm{d}, J=6.5), 7.22-7.42(9 \mathrm{H}, \mathrm{m}) ;{ }^{13} \mathrm{C} \mathrm{NMR}\left(101 \mathrm{MHz} ; \mathrm{CDCl}_{3}\right) \delta 34.9,60.3$, $93.4,112.8,118.9,120.3,127.0,127.5,127.7,128.8,128.8,128.9,129.2,129.3$, 135.4, 136.8, 141.9; IR (neat) 3392, 3030, 2916, 1595, $1550 \mathrm{~cm}^{-1}$; Mass Spec (ES, M + H) Theoretical: 367.1213, Measured: 367.1214.

\section{$\left(2 R^{*}, 3 S^{*}\right)$-2-chloro-N-(3-(4-methylphenyl)-2-nitro-1-phenylpropyl)aniline (9c)}

White solid, mp 98-100 ${ }^{\circ} \mathrm{C} ;{ }^{1} \mathrm{H}$ NMR $\left(400 \mathrm{MHz} ; \mathrm{CDCl}_{3}\right) \delta 2.31(3 \mathrm{H}, \mathrm{s}), 3.19(1 \mathrm{H}, \mathrm{dd}$, $J=14.8,3.5), 3.42(1 \mathrm{H}, \mathrm{dd}, J=14.8,10.3), 5.03(1 \mathrm{H}, \mathrm{dd}, J=7.0,5.8), 5.09-5.16(1 \mathrm{H}, \mathrm{m})$, $5.30(1 \mathrm{H}, \mathrm{d}, J=7.0), 6.47(1 \mathrm{H}, \mathrm{dd}, J=8.2,1.1), 6.66(1 \mathrm{H}, \mathrm{td}, J=7.7,1.3), 6.95-7.05(3 \mathrm{H}$, $\mathrm{m})$, 7.05-7.14 (2H, m), 7.25-7.42 (6H, m); $\left.{ }^{13} \mathrm{C} \mathrm{NMR} \mathrm{(101} \mathrm{MHz;} \mathrm{CDCl}_{3}\right) \delta$ 21.0, 34.6, 60.2 , 93.4, 112.7, 118.8, 120.2, 126.9, 127.7, 128.6, 128.8, 129.2, 129.3, 129.6, 132.2, 136.8, 137.1, 141.1; IR (neat) 3442, 3029, 2922, 1597, $1552 \mathrm{~cm}^{-1}$; Mass Spec (ES, M + H) Theoretical: 381.1370 Measured: 381.1370.

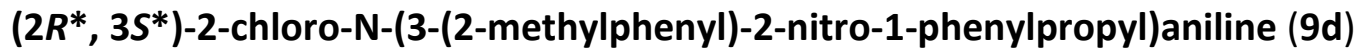

White solid, mp 117-118 ${ }^{\circ} \mathrm{C} ;{ }^{1} \mathrm{H}$ NMR $\left(400 \mathrm{MHz} ; \mathrm{CDCl}_{3}\right) \delta 2.11(3 \mathrm{H}, \mathrm{s}), 3.32(1 \mathrm{H}, \mathrm{dd}$, $J=15.2,2.6), 3.53(1 \mathrm{H}, \mathrm{dd}, J=15.2,10.4), 5.03-5.19(2 \mathrm{H}, \mathrm{m}), 5.32(1 \mathrm{H}, \mathrm{d}, J=6.3), 6.45-$ $6.57(1 \mathrm{H}, \mathrm{m}), 6.72(1 \mathrm{H}, \mathrm{td}, J=7.7,1.3), 6.99-7.08(1 \mathrm{H}, \mathrm{m}), 7.08-7.22(4 \mathrm{H}, \mathrm{m}), 7.30-7.52$ $(6 \mathrm{H}, \mathrm{m}) ;{ }^{13} \mathrm{C}$ NMR $\left(101 \mathrm{MHz} ; \mathrm{CDCl}_{3}\right) \delta 18.9,31.8,60.7,92.5,112.8,118.9,120.3$, $126.5,126.9,127.6,127.7,128.8,129.2$, 129.3, 129.3, 129.4, 133.6, 136.1, 136.9, 
141.9; IR (neat) 3403, 3064, 2925, 1596, $1552 \mathrm{~cm}^{-1}$; Mass Spec (ES, $M+H$ ) Theoretical: 381.1370 Measured: 381.1366.

$\left(2 R^{*}, 3 S^{*}\right)$-2-chloro-N-(3-(4-methoxyphenyl)-2-nitro-1-phenylpropyl)aniline (9e)

White solid, mp 73-75 ${ }^{\circ} \mathrm{C} ;{ }^{1} \mathrm{H}$ NMR (400 MHz; $\left.\mathrm{CDCl}_{3}\right) \delta 3.18(1 \mathrm{H}, \mathrm{dd}, J=14.8,3.5), 3.41$ $(1 \mathrm{H}, \mathrm{dd}, J=14.8,10.3), 3.78(3 \mathrm{H}, \mathrm{s}), 4.96-5.06(1 \mathrm{H}, \mathrm{m}), 5.06-5.14(1 \mathrm{H}, \mathrm{m}), 5.28(1 \mathrm{H}, \mathrm{d}$, $J=7.0), 6.47(1 \mathrm{H}, \mathrm{dd}, J=8.3,1.0), 6.67(1 \mathrm{H}, \mathrm{td}, J=7.7,1.3), 6.78-6.89(2 \mathrm{H}, \mathrm{m}), 6.95-7.10$ $(3 \mathrm{H}, \mathrm{m}), 7.23-7.45(6 \mathrm{H}, \mathrm{m}) ;{ }^{13} \mathrm{C} \mathrm{NMR}\left(101 \mathrm{MHz} ; \mathrm{CDCl}_{3}\right) \delta 34.2,55.3,60.2,93.6,112.8$, $114.4,118.8,120.2,126.9,127.2,127.7,128.8,129.2,129.3,129.9,136.8,141.9$, 159.0; IR (neat) 3402, 3032, 2933, 1596, $1551 \mathrm{~cm}^{-1}$; Mass Spec (ES, M + H) Theoretical: 397.1319 Measured: 397.1314.

$\left(2 R^{*}, 3 S^{*}\right)$-2-chloro-N-(3-(2-methoxyphenyl)-2-nitro-1-phenylpropyl)aniline (9f)

White solid, $\mathrm{mp} \mathrm{90-92}{ }^{\circ} \mathrm{C} ;{ }^{1} \mathrm{H}$ NMR $\left(400 \mathrm{MHz} ; \mathrm{CDCl}_{3}\right) \delta 3.28(1 \mathrm{H}, \mathrm{dd}, \mathrm{J}=14.4,9.9), 3.45$ $(1 \mathrm{H}, \mathrm{dd}, J=14.4,3.1), 3.75(3 \mathrm{H}, \mathrm{s}), 5.02(1 \mathrm{H}$, app t, J=6.5), 5.21 (1H, ddd, J=9.9, 6.1, 3.2), $5.35(1 \mathrm{H}, \mathrm{d}, J=6.8), 6.47(1 \mathrm{H}, \mathrm{dd}, J=8.0,1.0), 6.65$ (1H, td, $J=7.7,1.5), 6.79-6.90$ $(2 \mathrm{H}, \mathrm{m}), \quad 6.94-7.03(1 \mathrm{H}, \mathrm{m}), 7.10(1 \mathrm{H}, \mathrm{dd}, J=7.7,1.5), 7.18-7.43(7 \mathrm{H}, \mathrm{m}) ;{ }^{13} \mathrm{C} N M R$ $\left(101 \mathrm{MHz} ; \mathrm{CDCl}_{3}\right) \delta 31.0,55.2,60.4,91.6,110.4,112.6,118.5,120.0,120.8,123.7$, $127.0,127.7,128.6,128.9,129.0,129.2$, 131.0, 137.0, 142.0, 157.2; IR (neat) 3403, 3031, 2939, 1596, $1550 \mathrm{~cm}^{-1}$; Mass Spec (ES, $M+H$ ) Theoretical: 397.1319 Measured: 397.1316.

\section{$\left(2 R^{*}, 3 S^{*}\right)$-2-chloro-N-(2-nitro-1-phenyl-3-(pyridin-3-yl)propyl)aniline (9g)}

Yellow oil; ${ }^{1} \mathrm{H}$ NMR (400 MHz; $\left.\mathrm{CDCl}_{3}\right) \delta 3.23(1 \mathrm{H}, \mathrm{dd}, \mathrm{J}=15.1,2.5), 3.47$ (1H, dd, J=15.1, 10.2), 5.02-5.16 (2H, m), $5.22(1 \mathrm{H}, \mathrm{d}, J=6.8), 6.50(1 \mathrm{H}, \mathrm{dd}, J=8.3,1.3), 6.68(1 \mathrm{H}, \mathrm{td}$, $J=7.7,1.5), 6.94-7.08(1 \mathrm{H}, \mathrm{m}), 7.20(1 \mathrm{H}, \mathrm{dd}, J=7.6,4.8), 7.28$ (1H, dd, J=7.9, 1.4), 7.31 - $7.47(6 \mathrm{H}, \mathrm{m}), 8.40(1 \mathrm{H}, \mathrm{d}, J=2.0), 8.50(1 \mathrm{H}, \mathrm{dd}, J=4.8,1.5) ;{ }^{13} \mathrm{C} \mathrm{NMR}\left(101 \mathrm{MHz} ; \mathrm{CDCl}_{3}\right)$ $\delta 32.3,60.4,92.9,112.8,119.1,120.4,123.8,126.8,127.8,129.0,129.4,129.4$, 131.5, 136.5, 136.9, 141.7, 148.4, 149.7; IR (neat) 3400, 3031, 1596, $1552 \mathrm{~cm}^{-1}$; Mass Spec (ES, M + H) Theoretical: 367.1166 Measured: 367.1165.

\section{$\left(2 R^{*}, 3 S^{*}\right)$-4-(tert-butyl)-2-chloro-N-(2-nitro-1,3-diphenylpropyl)aniline (9h)}

White solid, mp 125-127 ${ }^{\circ} \mathrm{C} ;{ }^{1} \mathrm{H}$ NMR (400 MHz; $\left.\mathrm{CDCl}_{3}\right) \delta 1.24\left(9 \mathrm{H}, \mathrm{s}, \mathrm{Bu}^{\mathrm{t}}\right), 3.24(1 \mathrm{H}$, dd, J=14.9, 3.4), $3.47(1 \mathrm{H}, \mathrm{dd}, J=14.8,10.5), 5.03(1 \mathrm{H}, \mathrm{dd}, J=6.8,5.8), 5.08-5.18(2 \mathrm{H}$, $m,), 6.44(1 \mathrm{H}, d, J=8.5), 7.03(1 \mathrm{H}, \mathrm{dd}, J=8.5$ and 2.3$), 7.08-7.16(2 \mathrm{H}, \mathrm{m}), 7.22-7.41(9 \mathrm{H}$, 
m); ${ }^{13} \mathrm{C}$ NMR (101 MHz; CDCl3) $\delta 31.3,34.0,35.0,60.6,93.6,112.5,120.0,124.6$, $126.4,126.9,127.5,128.8,128.9,129.2$, 135.5, 137.1, 139.5, 142.2; IR (neat) 3404, 3032, 2962, 1611, $1551 \mathrm{~cm}^{-1}$; Mass Spec (ES, $M+H$ ) Theoretical: 423.1839 Measured: 423.1833.

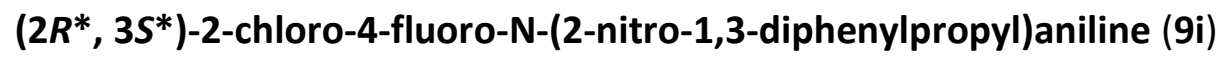
White solid, mp 109-112 ${ }^{\circ} \mathrm{C} ;{ }^{1} \mathrm{H}$ NMR (400 MHz; $\left.\mathrm{CDCl}_{3}\right) \delta 3.21(1 \mathrm{H}, \mathrm{dd}, J=14.9,3.6)$, $3.46(1 \mathrm{H}, \mathrm{dd}, J=14.9,10.4), 4.99(1 \mathrm{H}, \mathrm{dd}, J=6.8,5.5), 5.09-5.20(2 \mathrm{H}, \mathrm{m}), 6.37(1 \mathrm{H}, \mathrm{dd}$, $J=6.8,5.5), 6.73(1 \mathrm{H}, \mathrm{td}, J=8.5,2.9), 7.01(1 \mathrm{H}, \mathrm{dd}, J=8.5$ and 2.3), 7.09-7.16 $(2 \mathrm{H}, \mathrm{m})$, 7.21-7.44 (8H, m); ${ }^{13} \mathrm{C}$ NMR (101 MHz; $\left.\mathrm{CDCl}_{3}\right) \delta 34.8,60.8,93.2,113.2\left(\mathrm{~d}, J_{C F}=8.0\right.$ ), $114.4\left(d, J_{C F}=22.4\right), 116.7\left(d, J_{C F}=25.6\right), 120.3\left(d, J_{C F}=10.4\right), 126.8,127.5,128.8,128.9$, 129.3, 135.3, 136.5, 138.6 (d, $\left.J_{C F}=3.2\right), 155.2$ (d, $\left.J_{C F}=240.5\right)$; ${ }^{19} \mathrm{~F} \mathrm{NMR}\left(376 \mathrm{MHz}, \mathrm{CDCl}_{3}\right.$ ) ppm -63.11 (s); IR (neat) 3405, 3031, 1552, $1509 \mathrm{~cm}^{-1}$; Mass Spec (ES, M + H) Theoretical: 385.1119 Measured: 385.1119.

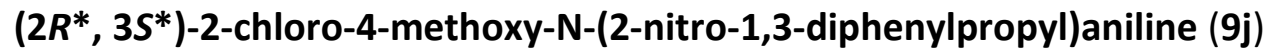

White solid, mp 125-127 ${ }^{\circ} \mathrm{C} ;{ }^{1} \mathrm{H}$ NMR (400 MHz; $\left.\mathrm{CDCl}_{3}\right) \delta 3.23(1 \mathrm{H}, \mathrm{dd}, \mathrm{J}=14.8,3.5)$, $3.46(1 \mathrm{H}, \mathrm{dd}, J=14.8,10.3), 3.64(3 \mathrm{H}, \mathrm{s}), 5.01(1 \mathrm{H}, \mathrm{dd}, J=7.0,5.8), 5.14(1 \mathrm{H}, \mathrm{ddd}$, $J=10.2,5.7,3.5), 5.27(1 \mathrm{H}, \mathrm{d}, J=7.0), 6.04(1 \mathrm{H}, \mathrm{d}, J=2.8), 6.23(1 \mathrm{H}, \mathrm{dd}, J=8.5,2.8), 7.13$ $(2 \mathrm{H}, \mathrm{d}, J=6.8), 7.17(1 \mathrm{H}, \mathrm{d}, J=8.5), 7.23-7.32(3 \mathrm{H}, \mathrm{m}), 7.33-7.43(5 \mathrm{H}, \mathrm{m}) ;{ }^{13} \mathrm{C}$ NMR $(101$ $\mathrm{MHz} ; \mathrm{CDCl} 3) \delta 34.9,55.3,60.3,93.3,99.8,103.4,112.2,126.8,127.5,128.8,128.9$, $128.9,129.3,129.5,129.3,135.3,136.6,142.6,159.4$; IR (neat) 3402, 3031, 2937, 1602, $1553 \mathrm{~cm}^{-1}$; Mass Spec (ES, M + H) Theoretical: 397.1319 Measured: 397.1330.

\section{$\left(2 R^{*}, 3 S^{*}\right)$-2-chloro-N-(2-nitro-1,3-diphenylpropyl)-5-(trifluoromethyl)aniline (9I)}

Oil; ${ }^{1} \mathrm{H}$ NMR (400 MHz; $\mathrm{CDCl}_{3}$ ) $\delta 3.18(1 \mathrm{H}, \mathrm{dd}, J=14.8,4.0), 3.43$ (1H, dd, $J=14.8,10.3$ ), $5.02(1 \mathrm{H}, \mathrm{dd}, J=6.9,5.4), 5.15$ (1H, ddd, $J=10.3,5.1,3.8), 5.52(1 \mathrm{H}, \mathrm{d}, J=7.0), 6.59-6.64$ $(1 \mathrm{H}, \mathrm{m}), 6.90(1 \mathrm{H}, \mathrm{dd}, J=8.3,1.3), 7.08-7.16(2 \mathrm{H}, \mathrm{m}), 7.19-7.45(9 \mathrm{H}, \mathrm{m}) ;{ }^{13} \mathrm{C} N M R$ (101 MHz; $\left.\mathrm{CDCl}_{3}\right) \delta$ 34.5, 59.7, 92.4, 108.7 ( $\left.\mathrm{Ar}, \mathrm{J}=4.0\right), 115.0(\mathrm{Ar}, \mathrm{J}=4.0), 123.2$ (q, $J=1.6$ ), 123.3 (q, J=272.4), 126.5, 127.3, 128.5, 128.7, 128.9, 129.1, 129.3, 129.9 (q, $J=32.8), 134.7,135.3,141.8 ;{ }^{19} \mathrm{~F} \mathrm{NMR}\left(376 \mathrm{MHz}, \mathrm{CDCl}_{3}\right.$ ) $\delta-63.4$; IR (neat) 3402, 3066, 3032, 1604, $1552 \mathrm{~cm}^{-1}$; Mass Spec (ES, M + H) Theoretical: 435.1087 Measured: 435.1080 .

$\left(2 R^{*}, 3 S^{*}\right)-2$-chloro-N-(2-nitro-1,3-diphenylpropyl)pyridin-3-amine (9m) 
White solid, $\mathrm{mp} 142-144{ }^{\circ} \mathrm{C} ;{ }^{1} \mathrm{H}$ NMR $\left(400 \mathrm{MHz} ; \mathrm{CDCl}_{3}\right) \delta 3.18(1 \mathrm{H}, \mathrm{dd}, J=14.8,3.8)$, 3.45 (1H, dd, J=14.8, 10.3), 4.93-5.04 (1H, m), 5.16 (1H, ddd, J=10.3, 5.1, 3.8), 5.41 $(1 \mathrm{H}, \mathrm{d}, J=6.8), 6.65(1 \mathrm{H}, \mathrm{dd}, J=8.0,1.3), 6.94(1 \mathrm{H}, \mathrm{dd}, J=8.0,4.8), 7.11(2 \mathrm{H}, \mathrm{d}, J=6.5)$, 7.19 - $7.45(8 \mathrm{H}, \mathrm{m}), 7.74(1 \mathrm{H}, \mathrm{dd}, J=4.5,1.5) ;{ }^{13} \mathrm{C} \mathrm{NMR}\left(101 \mathrm{MHz} ; \mathrm{CDCl}_{3}\right) \delta 34.7,59.9$, $92.9,119.2,123.2,126.8,126.8,127.6,128.8,129.1$, 129.4, 135.0, 135.7, 138.0, 138.7; IR (neat) 3401, 3032, 1583, $1551 \mathrm{~cm}^{-1}$; Mass Spec (ES, M + H) Theoretical: 367.1166 Measured: 367.1158.

\section{$\left(2 R^{*}, 3 S^{*}\right)$-2-chloro-N-(1-(3-methoxyphenyl)-2-nitro-3-phenylpropyl)aniline (9n)}

White solid, mp 88-90 ${ }^{\circ} \mathrm{C} ;{ }^{1} \mathrm{H}$ NMR (400 MHz; CDCl3) $\delta 3.20(1 \mathrm{H}, \mathrm{dd}, J=14.8,3.5), 3.44$ $(1 \mathrm{H}, \mathrm{dd}, J=14.8,10.5), 3.78(3 \mathrm{H}, \mathrm{s}), 4.95-5.04(1 \mathrm{H}, \mathrm{m}), 5.07-5.15(1 \mathrm{H}, \mathrm{m}), 5.24(1 \mathrm{H}, \mathrm{d}$, $J=7.0), 6.45(1 \mathrm{H}, \mathrm{dd}, J=8.3,1.3), 6.66(1 \mathrm{H}, \mathrm{td}, J=7.7,1.3), 6.83-6.90(2 \mathrm{H}, \mathrm{m}), 6.93-7.03$ $(2 \mathrm{H}, \mathrm{m}), 7.07-7.13(2 \mathrm{H}, \mathrm{m}), 7.22-7.32(5 \mathrm{H}, \mathrm{m}) ;{ }^{13} \mathrm{C} \mathrm{NMR} \mathrm{(101} \mathrm{MHz;} \mathrm{CDCl3)} \delta 34.8$, $55.3,60.3,93.3,112.8,112.8,113.9,118.9,119.0,120.3,127.5,127.7,128.8,128.9$, 129.3, 130.3, 135.4, 138.5, 141.9, 160.2; IR (neat) 3404, 3030, 2936, 1596, $1551 \mathrm{~cm}^{-1}$; Mass Spec (ES, M + H) Theoretical: 397.1319 Measured: 397.1330.

$\left(2 R^{*}\right.$,

$\left.3 S^{*}\right)$-2-chloro-N-(2-nitro-3-phenyl-1-(3-

(trifluoromethyl)phenyl)propyl)aniline (9o)

Colourless oil; ${ }^{1} \mathrm{H}$ NMR (400 MHz; $\mathrm{CDCl}_{3}$ ) for major isomer $\delta 3.26(1 \mathrm{H}, \mathrm{dd}, J=14.8$, 3.8,), $3.51(1 \mathrm{H}, \mathrm{dd}, J=14.8,10.0), 5.09-5.15(1 \mathrm{H}, \mathrm{m}), 5.21(1 \mathrm{H}, \mathrm{ddd}, J=10.0,5.8,3.8)$, $5.35(1 \mathrm{H}, \mathrm{d}, J=7.03), 6.46(1 \mathrm{H}, \mathrm{dd}, J=8.2,1.1), 6.74(1 \mathrm{H}, \mathrm{td}, J=7.7,1.4), 7.03-7.09(1 \mathrm{H}$, m), 7.14-7.20 $(2 \mathrm{H}, \mathrm{m}), 7.26-7.38(4 \mathrm{H}, \mathrm{m}), 7.49-7.71(4 \mathrm{H}, \mathrm{m}) ;{ }^{13} \mathrm{C}$ NMR for major isomer $\delta\left(101 \mathrm{MHz} ; \mathrm{CDCl}_{3}\right)$ 35.1, 59.9, 92.8, 112.7, 119.3, 120.4, 131.6 (q, J=272.2 Hz), 123.8 (q, J=3.7), 125.8 (q, J=3.5), 127.7, 127.8, 128.8, 129.0, 129.5, 129.8, 130.2, 131.6 (q, J=32.5 Hz), 134.8, 138.1, 141.4; IR (neat) 3403, 3032, 2926, 1596, $1554 \mathrm{~cm}^{-}$ 1; Mass Spec (ES, M + H) Theoretical: 435.1087 Measured: 435.1094.

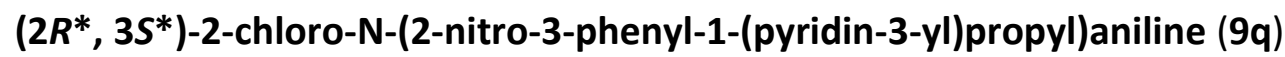

Yellow oil; ${ }^{1} \mathrm{H}$ NMR (400 MHz; $\left.\mathrm{CDCl}_{3}\right) \delta 3.27\left(1 \mathrm{H}, \mathrm{dd}, J=14.6,4.0, \mathrm{CH}_{\mathrm{a}}\right), 3.46(1 \mathrm{H}, \mathrm{dd}$, $\left.J=14.6,9.0, \mathrm{CH}_{\mathrm{b}}\right), 4.90-5.08\left(3 \mathrm{H}, \mathrm{m}, J=9.5,5.8, \mathrm{CHNH}, \mathrm{CHNO}_{2}\right.$ and $\left.\mathrm{NH}\right), 6.37(1 \mathrm{H}, \mathrm{d}$, $J=1.0, \operatorname{ArH}), 6.57(1 \mathrm{H}, \mathrm{d}, J=8.2, \operatorname{ArH}), 6.70(1 \mathrm{H}, \mathrm{dt}, J=7.7,1.3, \operatorname{ArH}), 7.03-7.11(1 \mathrm{H}, \mathrm{m}$, $\operatorname{Ar} H)$, 7.13-7.20 (2H, m, ArH), 7.23-7.35 (4H, m, ArH), 7.39-7.45 (2H, m, ArH); ${ }^{13} \mathrm{C} \mathrm{NMR}$ $\left(101 \mathrm{MHz} ; \mathrm{CDCl}_{3}\right) \delta 35.7,52.7,92.2,108.3,112.6,119.2,120.3,121.9,127.6,127.8$, 
$128.9,129.0,129.5,135.2,140.7,141.8,144.3$; IR (neat) 3405, 3030, 2936, 1597, $1554 \mathrm{~cm}^{-1}$; Mass Spec (ES, M + H) Theoretical: 357.1006 Measured: 357.0997.

\section{$\left(2 R^{*}, 3 S^{*}\right)$-2-chloro-N-(2-nitro-3-phenyl-1-(pyridin-3-yl)propyl)aniline (9r)}

White solid, mp 101-103 ${ }^{\circ} \mathrm{C} ;{ }^{1} \mathrm{H}$ NMR (400 MHz; $\left.\mathrm{CDCl}_{3}\right) \delta 3.26(1 \mathrm{H}, \mathrm{dd}, \mathrm{J}=14.8,4.0)$, $3.45(1 \mathrm{H}, \mathrm{dd}, J=14.8,9.8), 4.99-5.08(1 \mathrm{H}, \mathrm{m}), 5.13-5.23(1 \mathrm{H}, \mathrm{m}), 5.29(1 \mathrm{H}, \mathrm{d}, J=8.0)$, $6.44(1 \mathrm{H}, \mathrm{d}, J=8.0), 6.64-6.75(1 \mathrm{H}, \mathrm{m}), 6.94-7.07(1 \mathrm{H}, \mathrm{m}), 7.16(2 \mathrm{H}, \mathrm{d}, J=6.5), 7.27-$ $7.35(5 \mathrm{H}, \mathrm{m}), 7.62-7.70(1 \mathrm{H}, \mathrm{m}), 8.61(1 \mathrm{H}, \mathrm{dd}, J=4.8,1.5), 8.68(1 \mathrm{H}, \mathrm{d}, J=2.3) ;{ }^{13} \mathrm{C}$ NMR $\left(101 \mathrm{MHz} ; \mathrm{CDCl}_{3}\right) \delta 35.5,58.0,92.7,112.7,119.4,120.4,124.0,127.7,127.8$, $128.8,129.0,129.5,132.4,134.3,134.7,141.2,148.9,150.3$; IR (neat) 3400, 3033, 1595, $1553 \mathrm{~cm}^{-1}$; Mass Spec (ES, M + H) Theoretical: 367.1166 Measured: 367.1171.

General Procedure for the Synthesis of 1,2,3,4-tetrahydroquinoxalines 7 (Table 3) To a solution of $\beta$-nitroamine 9 (3.0 mmol 1.0 eq.) in EtOH $(50 \mathrm{~mL}$ ) was added Zinc (24.0 mmol, 8 equiv) follow by hydrochloric acid (60.0 mmol, 6M, 20 eq.). The reaction mixture was stirred at $\mathrm{rt}$ for $1 \mathrm{~h}$ and the solvent removed in vacuo. A saturated aq. solution of $\mathrm{NaHCO}_{3}(20 \mathrm{~mL})$ was added and the mixture was extracted with EtOAc $(3 \times 20 \mathrm{~mL})$, the combined organics washed with brine $(10 \mathrm{~mL})$, dried $\left(\mathrm{MgSO}_{4}\right)$ and the solvent removed in vacuo to give crude 1,2-diamine 8.

A solution of the crude 1,2-diamine 8 in $\mathrm{PhMe}(1.5 \mathrm{~mL})$ was added to ${ }^{\mathrm{t}} \mathrm{BuONa}(1.00$ mmol, 2.0 equiv), X-Phos ( $0.040 \mathrm{mmol}, 8 \mathrm{~mol} \%)$ and palladium(II) acetate $(0.020$ $\mathrm{mmol}, 4 \mathrm{~mol} \%)$ in PhMe $(1 \mathrm{~mL})$ under $\mathrm{N}_{2}$ at rt. The reaction was heated to $90{ }^{\circ} \mathrm{C}$ for $3-$ $12 \mathrm{hrs}$ until complete conversion. The reaction mixture was allowed to cool to rt, filtered thought celite and concentrated to give crude 1,2,3,4-tetrahydroquinoxaline 7 which was purified by column chromatography.

$\left(2 R^{*}, 3 S^{*}\right)$-2-benzyl-3-phenyl-1,2,3,4-tetrahydroquinoxaline (7b)

Yellow solid, mp 63-65 ${ }^{\circ} \mathrm{C} ;{ }^{1} \mathrm{H}$ NMR $\left(600 \mathrm{MHz} ; \mathrm{CDCl}_{3}\right) \delta 2.47(1 \mathrm{H}, \mathrm{dd}, J=13.5,10.7)$, $2.57(1 \mathrm{H}, \mathrm{dd}, J=13.5,3.3), 3.70(1 \mathrm{H}, \mathrm{s}), 3.73(1 \mathrm{H}, \mathrm{app} . \mathrm{dt}, J=10.7,3.3), 4.00(1 \mathrm{H}, \mathrm{s})$, $4.76(1 \mathrm{H}, \mathrm{d}, J=2.9), 6.47(1 \mathrm{H}, \mathrm{dd}, J=7.6,1.3), 6.59(1 \mathrm{H}, \mathrm{dd}, J=7.5,1.4), 6.63(1 \mathrm{H}, \mathrm{td}, J$ $=7.5,1.4), 6.67(1 \mathrm{H}, \mathrm{td}, J=7.4,1.3), 7.02-7.06(2 \mathrm{H}, \mathrm{m}), 7.19-7.23(1 \mathrm{H}, \mathrm{m}), 7.25-7.29$ $(2 \mathrm{H}, \mathrm{m}), 7.31-7.34(1 \mathrm{H}, \mathrm{m}), 7.36-7.43(4 \mathrm{H}, \mathrm{m}) ;{ }^{13} \mathrm{C} \mathrm{NMR}\left(151 \mathrm{MHz} ; \mathrm{CDCl}_{3}\right) \delta 36.5,55.7$, $58.1,114.3,115.0,118.9,119.2,126.5,127.6,127.7,128.6,128.7,128.5,132.1$, 
133.4, 138.8, 141.6; IR (neat) 3384, 3060, 3025, 1550, $1504 \mathrm{~cm}^{-1}$; Mass Spec (EI) Theoretical: 300.1621 Measured: 300.1627.

\section{$\left(2 R^{*}, 3 S^{*}\right)-2-(4-m e t h y l b e n z y l)-3-p h e n y l-1,2,3,4-t e t r a h y d r o q u i n o x a l i n e ~(7 c)$}

Yellow solid, mp 63-65 ${ }^{\circ} \mathrm{C} ;{ }^{1} \mathrm{H}$ NMR $\left(400 \mathrm{MHz} ; \mathrm{CDCl}_{3}\right) \delta 2.36(3 \mathrm{H}, \mathrm{s}), 2.47(1 \mathrm{H}, \mathrm{dd}, J$ $=13.3,10.5), 2.57(1 \mathrm{H}, \mathrm{dd}, J=13.3,3.5), 3.75$ (1H, app. dt, $J=10.4,3.5), 3.93$ (2H, br. s), $4.77(1 \mathrm{H}, \mathrm{d}, J=3.0), 6.50(1 \mathrm{H}, \mathrm{d}, J=7.3,1.5), 6.57-6.76(3 \mathrm{H}, \mathrm{m}), 6.93-7.01(2 \mathrm{H}, \mathrm{m})$, 7.06-7.17 (2H, m), 7.30-7.48 (5H, m); ${ }^{13} \mathrm{C}$ NMR (101 MHz; $\left.\mathrm{CDCl}_{3}\right) \delta$ 21.0, 36.0, 55.6, 58.0, 114.2, 114.8, 118.7, 119.1, 127.5, 127.5, 128.4, 129.2, 129.3, 132.1, 133.3, $135.5,135.9,141.7$; IR (neat) 3376, 3023, 2920, $1508 \mathrm{~cm}^{-1}$; Mass Spec (ES, M + H) Theoretical: 315.1861 Measured: 315.1855.

$\left(2 R^{*}, 3 S^{*}\right)-2-(2-m e t h y l b e n z y l)-3-p h e n y l-1,2,3,4-t e t r a h y d r o q u i n o x a l i n e ~(7 d)$

Yellow solid, mp 58-60 ${ }^{\circ} \mathrm{C} ;{ }^{1} \mathrm{H}$ NMR $\left(400 \mathrm{MHz} ; \mathrm{CDCl}_{3}\right) \delta 2.00(3 \mathrm{H}, \mathrm{s}), 2.53(1 \mathrm{H}, \mathrm{dd}, J$ $=13.7,10.3), 2.61(1 \mathrm{H}, \mathrm{dd}, J=13.7,3.3), 3.69$ (1H, app. dt, $J=10.4,3.1), 3.89(2 \mathrm{H}, \mathrm{br}$. s), $4.83(1 \mathrm{H}, \mathrm{d}, J=3.0), 6.50(1 \mathrm{H}, \mathrm{d}, J=7.0,1.8), 6.59-6.71(3 \mathrm{H}, \mathrm{m}), 6.93-7.01(1 \mathrm{H}, \mathrm{m})$, 7.09-7.15 (3H, m), 7.33-7.50 (5H, m); $\left.{ }^{13} \mathrm{C} \mathrm{NMR} \mathrm{(101} \mathrm{MHz;} \mathrm{CDCl}_{3}\right) \delta$ 18.7, 32.7, 54.2, 57.7, 114.1, 114.6, 118.7, 125.6, 126.2, 127.0, 127.3, 128.1, 129.8, 130.2, 131.8, 132.9, 136.4, 136.7, 141.1; IR (neat) 3368, 3022, 2925, $1505 \mathrm{~cm}^{-1}$; Mass Spec (ES, M + H) Theoretical: 315.1861 Measured: 315.1854.

$\left(2 R^{*}, 3 S^{*}\right)-2-(4-m e t h o x y b e n z y l)-3-p h e n y l-1,2,3,4-t e t r a h y d r o q u i n o x a l i n e ~(7 e)$ Yellow solid, mp 58-60 ${ }^{\circ} \mathrm{C} ;{ }^{1} \mathrm{H}$ NMR $\left(400 \mathrm{MHz} ; \mathrm{CDCl}_{3}\right) \delta 2.45(1 \mathrm{H}, \mathrm{dd}, J=13.7,10.7)$, $2.55(1 \mathrm{H}, \mathrm{dd}, J=13.7,3.6), 3.73(1 \mathrm{H}, \mathrm{app} . \mathrm{dt}, J=10.5,3.4), 3.82(3 \mathrm{H}, \mathrm{s}), 3.86(2 \mathrm{H}, \mathrm{br}$. s), $4.76(1 \mathrm{H}, \mathrm{d}, J=3.0), 6.51(1 \mathrm{H}, \mathrm{d}, J=7.4,1.6), 6.59-6.75(3 \mathrm{H}, \mathrm{m}), 6.80-6.90(2 \mathrm{H}, \mathrm{m})$, 6.95-7.03 (2H, m), 7.32-7.45 (5H, m); ${ }^{13} \mathrm{C}$ NMR (101 MHz; $\left.\mathrm{CDCl}_{3}\right) \delta 35.5,55.2,55.6$, 57.9, 114.0, 114.2, 115.0, 118.7, 119.3, 127.5, 127.6, 128.4, 130.3, 130.4, 131.8, 133.3, 141.5, 158.3; IR (neat) 3373, 3027, 2950, $1510 \mathrm{~cm}^{-1}$; Mass Spec (ES, M + H) Theoretical: 331.1810 Measured: 331.1807.

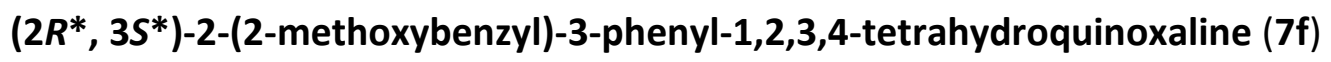
Yellow solid, mp 55-57 ${ }^{\circ} \mathrm{C} ;{ }^{1} \mathrm{H}$ NMR $\left(400 \mathrm{MHz} ; \mathrm{CDCl}_{3}\right) \delta 2.43(1 \mathrm{H}, \mathrm{dd}, J=13.3,10.3)$, $2.78(1 \mathrm{H}, \mathrm{dd}, J=13.3,3.0), 3.76(3 \mathrm{H}, \mathrm{s}), 3.82(2 \mathrm{H}, \mathrm{br} . \mathrm{s}), 3.91(1 \mathrm{H}, \mathrm{app} . \mathrm{dt}, J=10.1$, 3.0), $4.80(1 \mathrm{H}, \mathrm{d}, J=3.0), 6.54(1 \mathrm{H}, \mathrm{d}, J=7.3,1.5), 6.61-6.77(3 \mathrm{H}, \mathrm{m}), 6.84-6.97(2 \mathrm{H}$, m), $7.02(1 \mathrm{H}, \mathrm{dd}, J=7.3,1.5), 7.22-7.31(1 \mathrm{H}, \mathrm{m}), 7.35-7.55(5 \mathrm{H}, \mathrm{m}) ;{ }^{13} \mathrm{C}$ NMR $(101$ 
$\left.\mathrm{MHz} ; \mathrm{CDCl}_{3}\right) \delta 32.0,53.5,55.1,58.1,110.4,114.1,114.8,118.5,118.9,120.4,127.1$, $127.3,127.5,127.8,128.2,131.2$, 132.5, 133.2, 141.8, 157.8; IR (neat) 3374, 3027, 2949, $1510 \mathrm{~cm}^{-1}$; Mass Spec (ES, M + H) Theoretical: 331.1810 Measured: 331.1802.

\section{$\left(2 R^{*}, 3 S^{*}\right)$-3-benzyl-6-tert-butyl-2-phenyl-1,2,3,4-tetrahydroquinoxaline (7h)}

Yellow solid, mp 64-65 ${ }^{\circ} \mathrm{C} ;{ }^{1} \mathrm{H}$ NMR (400 MHz; CDCl $) \delta 1.30(9 \mathrm{H}, \mathrm{s}), 2.50(1 \mathrm{H}, \mathrm{dd}, J$ $=13.6,10.5), 2.59(1 \mathrm{H}, \mathrm{dd}, J=13.6,3.5), 3.76(1 \mathrm{H}, \mathrm{app} . \mathrm{dt}, J=10.5,3.3), 3.78(2 \mathrm{H}, \mathrm{br}$ s), $4.74(1 \mathrm{H}, \mathrm{d}, J=3.0), 6.48-6.58(2 \mathrm{H}, \mathrm{m}), 6.71(1 \mathrm{H}, \mathrm{dd}, J=8.3,2.0), 7.06(2 \mathrm{H}, \mathrm{d}, J$ =7.0), 7.02-7.44 (8H, m); ${ }^{13} \mathrm{C}$ NMR $\left(101 \mathrm{MHz} \mathrm{CDCl}_{3}\right) \delta$ 31.6, 33.9, 36.5, 55.8, 58.2, $112.3,113.8,115.9,126.3,127.5,127.5,128.4,128.5,129.4,130.8,131.5,138.8$, 141.8, 141.9; IR (neat) 3379, 3026, 2959, $1521 \mathrm{~cm}^{-1}$; Mass Spec (ES, M + H) Theoretical: 357.2331 Measured: 357.2318.

\section{$\left(2 R^{*}, 3 S^{*}\right)$-3-benzyl-6-fluoro-2-phenyl-1,2,3,4-tetrahydroquinoxaline (7i)}

Yellow solid, mp 53-55 ${ }^{\circ} \mathrm{C} ;{ }^{1} \mathrm{H}$ NMR $\left(400 \mathrm{MHz} ; \mathrm{CDCl}_{3}\right) \delta 2.50-2.64(2 \mathrm{H}, \mathrm{m}), 3.75(1 \mathrm{H}$, $\mathrm{td}, J=9.8,3.8), 3.84(2 \mathrm{H}, \mathrm{br}$ s), $4.75(1 \mathrm{H}, \mathrm{d}, J=3.0), 6.23(1 \mathrm{H}, \mathrm{dd}, J=10.0,2.5), 6.39$ $(1 \mathrm{H}, \mathrm{td}, J=8.5,2.8), 6.52(1 \mathrm{H}, \mathrm{dd}, J=8.5,5.3), 7.07(2 \mathrm{H}, \mathrm{d}, J=7.0), 7.20-7.50(8 \mathrm{H}, \mathrm{m})$; $\left.{ }^{13} \mathrm{C} \mathrm{NMR} \mathrm{(101} \mathrm{MHz;} \mathrm{CDCl}_{3}\right) \delta 36.2,55.8,57.8,101.5$ (d, J JF=26.4), 104.5 (d, J J $=22.4$ ), $114.5\left(\mathrm{~d}, J_{C F}=8.8\right), 126.5,127.3,127.7,128.5,128.6,129.1,129.4,133.0$ (d, $\left.J_{C F}=10.4\right)$, 138.6, 141.2, 156.9 (d, $J_{C F}=234.1$ ); $\left.{ }^{19} \mathrm{~F} \mathrm{NMR} \mathrm{(376} \mathrm{MHz,} \mathrm{CDCl}_{3}\right) \delta-125.9$ (s); IR (neat) 3379, 3026, 2959, $1521 \mathrm{~cm}^{-1}$; Mass Spec (ES, M + H) Theoretical: 319.1611 Measured: 319.1602.

$\left(2 R^{*}, 3 S^{*}\right)$-3-benzyl-6-methoxy-2-phenyl-1,2,3,4-tetrahydroquinoxaline $(7 \mathrm{j})$ Yellow oil; ${ }^{1} \mathrm{H}$ NMR $\left(400 \mathrm{MHz} ; \mathrm{CDCl}_{3}\right) \delta 2.47(1 \mathrm{H}, \mathrm{dd}, J=13.6,10.5), 2.56(1 \mathrm{H}, \mathrm{dd}, J$ $=13.6,3.5), 3.67-3.75(1 \mathrm{H}, \mathrm{m}), 3.73(3 \mathrm{H}, \mathrm{s}), 4.74(1 \mathrm{H}, \mathrm{d}, J=3.0), 6.20-6.25(2 \mathrm{H}, \mathrm{m})$, $6.44(1 \mathrm{H}, \mathrm{d}, J=9.0), 7.04$ (2H, app. $d, J=7.0), 7.19-7.41(8 \mathrm{H}, \mathrm{m}) ;{ }^{13} \mathrm{C}$ NMR $(101 \mathrm{MHz}$; $\left.\mathrm{CDCl}_{3}\right) \delta 36.1,55.5,55.8,58.0,100.7,103.5,116.1,125.0,126.4,127.5$ (2C), 127.6, $128.4(2 \mathrm{C}), 128.6$ (2C), 129.3 (2C), 134.6, 138.5, 141.3, 153.9; IR (neat) 3369, 3027, 2932, $1517 \mathrm{~cm}^{-1}$; Mass Spec (ES, M + H) Theoretical: 331.1810 Measured: 331.1797.

$\left(2 R^{*}, 3 S^{*}\right)$-2-benzyl-3-phenyl-6-(trifluoromethyl)-1,2,3,4-tetrahydroquinoxaline (7I) White solid, mp 50-53 ${ }^{\circ} \mathrm{C} ;{ }^{1} \mathrm{H}$ NMR $\left(400 \mathrm{MHz} ; \mathrm{CDCl}_{3}\right) \delta 2.45(1 \mathrm{H}, \mathrm{dd}, J=13.4,10.7)$, $2.57(1 \mathrm{H}, \mathrm{dd}, J=13.4,3.5), 3.74(1 \mathrm{H}$, app dt, $J=10.6,3.4), 3.98(2 \mathrm{H}, \mathrm{br} \mathrm{s}), 4.74(1 \mathrm{H}, \mathrm{d}, J$ $=3.3), 6.43(1 \mathrm{H}, \mathrm{d}, J=8.3), 6.77-6.80(1 \mathrm{H}, \mathrm{m}), 6.86(1 \mathrm{H}, \mathrm{d}, J=8.0), 7.02(2 \mathrm{H}, \mathrm{d}, J=6.8)$, 
7.21-7.30 (3H, m,), 7.30-7.40 (5H, m); $\left.{ }^{13} \mathrm{C} \mathrm{NMR} \mathrm{(101} \mathrm{MHz;} \mathrm{CDCl}_{3}\right) \delta 36.5,55.5,57.6$, 110.6 (q, J=4.0), 113.6, 116.0 (q, $J=4.0$ ), 120.6 (q, $J=32.2$ ), 124.9 (q, $J=270.8$ ),126.6, $127.4,127.9,128.6,128.7,129.3,132.8,134.9,138.3,140.8 ;{ }^{19} \mathrm{~F}$ NMR (376 MHz, $\left.\mathrm{CDCl}_{3}\right) \delta-61.7(\mathrm{~s})$; IR (neat) 3390, 3029, 2853, $1491 \mathrm{~cm}^{-1}$; Mass Spec (ES, $\mathrm{M}+\mathrm{H}$ ) Theoretical: 369.1579 Measured: 369.1575.

\section{$\left(2 R^{*}, 3 S^{*}\right)$-3-benzyl-2-phenyl-1,2,3,4-tetrahydropyrido[2,3-b]pyrazine (7m)}

Yellow solid, mp 58-61 ${ }^{\circ} \mathrm{C} ;{ }^{1} \mathrm{H}$ NMR $\left(400 \mathrm{MHz} ; \mathrm{CDCl}_{3}\right) \delta 2.43(1 \mathrm{H}, \mathrm{dd}, J=13.4,10.7)$, $2.62(1 \mathrm{H}, \mathrm{dd}, J=13.4,3.3), 3.87-3.94(1 \mathrm{H}, \mathrm{m}), 4.03(1 \mathrm{H}, \mathrm{br} \mathrm{s}), 4.70(1 \mathrm{H}, \mathrm{d}, J=3.0), 4.81$ $(1 \mathrm{H}, \mathrm{s}), 6.56(1 \mathrm{H}, \mathrm{dd}, J=7.5,5.0), 6.74(1 \mathrm{H}, \mathrm{d}, J=6.8), 7.05(2 \mathrm{H}, \mathrm{d}, J=7.3), 7.19-7.43$ $(8 \mathrm{H}, \mathrm{m}), 7.56(1 \mathrm{H}, \mathrm{d}, J=4.3) ;{ }^{13} \mathrm{C} \mathrm{NMR}\left(101 \mathrm{MHz} ; \mathrm{CDCl}_{3}\right) \delta 37.3,55.5,57.5,114.4$, 118.6, 126.6, 127.5, 127.9, 128.5, 128.6, 128.7, 129.3, 137.2, 137.9, 140.7, 145.9; IR (neat) 3388, 3321, 3027, 2923, $1493 \mathrm{~cm}^{-1}$; Mass Spec (ES, M + H) Theoretical: 302.1657 Measured: 302.1646.

\section{$\left(2 R^{*}, 3 S^{*}\right)$-2-benzyl-3-(3-methoxyphenyl)-1,2,3,4-tetrahydroquinoxaline $(7 \mathrm{n})$}

Yellow oil; ${ }^{1} \mathrm{H}$ NMR $\left(400 \mathrm{MHz} ; \mathrm{CDCl}_{3}\right) \delta 2.54(1 \mathrm{H}, \mathrm{dd}, J=13.3,9.8), 2.60(1 \mathrm{H}, \mathrm{dd}, J$ $=13.3,4.3), 3.73(1 \mathrm{H}, \mathrm{m}), 3.83(3 \mathrm{H}, \mathrm{s}), 4.76(1 \mathrm{H}, \mathrm{d}, J=3.0), 6.48(1 \mathrm{H}, \mathrm{dd}, J=7.4,1.6)$, 6.59-6.71 (3H, m), $6.89(1 \mathrm{H}, \mathrm{dd}, J=8.0,2.3), 6.99-7.09(4 \mathrm{H}, \mathrm{m}), 7.21-7.35(4 \mathrm{H}, \mathrm{m})$; ${ }^{13} \mathrm{C}$ NMR $\left(101 \mathrm{MHz} ; \mathrm{CDCl}_{3}\right) \delta 36.2,55.2,55.8,57.8,112.7,113.2,114.3,114.9,118.9$, 119.0, 119.7, 126.4, 128.6, 129.4, 129.5, 131.9, 133.2, 138.8, 143.2, 159.7; IR (neat) 3372, 3025, 2947, $1504 \mathrm{~cm}^{-1}$; Mass Spec (ES, M + H) Theoretical: 331.1810 Measured: 331.1802.

$\left(2 R^{*}, 3 S^{*}\right)$-2-benzyl-3-(3-(trifluoromethyl)phenyl)-1,2,3,4-tetrahydroquinoxaline (7o)

Yellow solid, mp 49-51 ${ }^{\circ} \mathrm{C} ;{ }^{1} \mathrm{H}$ NMR (400 MHz; $\left.\mathrm{CDCl}_{3}\right) \delta 2.55(2 \mathrm{H}, \mathrm{d}, J=7.3), 3.87(1 \mathrm{H}$, $\mathrm{td}, J=7.3,3.0), 4.74(1 \mathrm{H}, \mathrm{d}, J=3.0), 5.65(2 \mathrm{H}, \mathrm{br} \mathrm{s}), 6.63(1 \mathrm{H}, \mathrm{dd}, J=7.3,1.3), 6.67-6.75$ $(2 \mathrm{H}, \mathrm{m}), 6.75-6.88(1 \mathrm{H}, \mathrm{m}), 7.00(2 \mathrm{H}, \mathrm{d}, J=6.8), 7.18-7.32(3 \mathrm{H}, \mathrm{m}), 7.40-7.55(1 \mathrm{H}, \mathrm{m})$, 7.58-7.69 (3H, m); ${ }^{13} \mathrm{C} \mathrm{NMR}\left(101 \mathrm{MHz} ; \mathrm{CDCl}_{3}\right) \delta 35.2,55.4,57.1,115.6,117.0,120.0$, $121.8,124.0$ ( $q, J=272.4$ ), 124.3 (q, J=3.5), 124.9 ( $q, J=3.5$ ), 127.0, 128.1, 128.6, 129.2, 129.3, 130.8 (q, J=32.8), 130.9, 132.9, 137.1, 141.0; $\left.{ }^{19} \mathrm{~F} \mathrm{NMR} \mathrm{(376;} \mathrm{CDCl}_{3}\right) \delta-63.0$ (s); IR (neat) 3371, 3028, $1505 \mathrm{~cm}^{-1}$; Mass Spec (ES, M + H) Theoretical: 369.1579 Measured: 369.1569. 
$\left(2 R^{*}, 3 S^{*}\right)$-2-benzyl-3-(pyridin-3-yl)-1,2,3,4-tetrahydroquinoxaline (7r)

Yellow solid, mp 55-58 ${ }^{\circ} \mathrm{C} ;{ }^{1} \mathrm{H}$ NMR $\left(400 \mathrm{MHz} ; \mathrm{CDCl}_{3}\right) \delta 2.40(1 \mathrm{H}, \mathrm{dd}, J=13.6,10.3)$, $2.60(1 \mathrm{H}, \mathrm{dd}, J=13.6,4.0), 3.72(1 \mathrm{H}, \mathrm{br} \mathrm{s}), 3.85(1 \mathrm{H}, \mathrm{app} \mathrm{dt}, J=10.1,3.6), 4.07(1 \mathrm{H}, \mathrm{br}$ s), $4.69(1 \mathrm{H}, \mathrm{d}, J=2.8), 6.41-6.54(1 \mathrm{H}, \mathrm{m}), 6.59(1 \mathrm{H}, \mathrm{dd}, J=7.3,1.5), 6.63-6.74(2 \mathrm{H}, \mathrm{m})$, $7.09(2 \mathrm{H}, \mathrm{d}, J=7.3), 7.24-7.36(4 \mathrm{H}, \mathrm{m}), 7.73(1 \mathrm{H}, \mathrm{d}, J=8.0), 8.55-8.64(2 \mathrm{H}, \mathrm{m}) ;{ }^{13} \mathrm{C}$ $\operatorname{NMR}\left(101 \mathrm{MHz} ; \mathrm{CDCl}_{3}\right) \delta 37.3,54.7,56.0,114.4,114.9,118.9,119.5,123.6,126.7$, $128.7,129.2$, 132.0, 132.5, 135.4, 137.2, 137.7, 149.0, 149.2; IR (neat) 3372, 3321, 3027, 2919, 1602, $1504 \mathrm{~cm}^{-1}$; Mass Spec (ES, $M+H$ ) Theoretical: 302.1657 Measured: 302.1658.

\section{Acknowledgements}

We thank UCL the EPSRC and GSK for funding (EP/F068344/2).

\section{References}

1) (a) C. T. Eary, Z. S.Jones, R. D. Groneberg, L. E. Burgess, D. A. Mareska, M. D. Drew, J. F. Blake, E. R. Laird, D. Balachari, M. O’Sullivan, A. Allen and V. Marsh, Bioorg. Med. Chem. Lett., 2007, 17, 2608. (b) Y. Ohtake, A. Naito, H. Hasegawa, K. Kawano, D. Marizono, M. Taniguchi, Y. Tanaka, H. Matsukawa, K. Naito, T. Oguma, Y. Ezure and, Y. Tsuriya, Bioorg. Med. Chem., 1999, 7, 1247. (c) K. Torisu, K. Kobayashi, M. Iwahashi, Y. Nakai, T. Onoda, I. Sugimoto, Y. Okada, R. Matsumoto, F. Nanbu, S. Ohuchida, H. Nakai and M. Toda, Bioorg. Med. Chem., 2004, 12, 5361. (d) J. A. Sikorski, J. Med. Chem., 2006, 49, 1. (e) E. J. Jacobsen, L. S. Stelzer, K. L. Belonga, D. B. Carter, W. B. Im, V. H. Sethy, A. H. Tang, P. F. Von-Voigtlander and J. D. Petke, J. Med. Chem., 1996, 39, 3820. (f) T. H. Barrows, P. R. Farina, R. L. Chrzanowski, P. A.; Benkovic, and S. J. Benkovic, J. Am. Chem. Soc., 1976, 98, 3678.

2) (a) E. Merişor, J. Conrad, I. Klaiber, S. Mika and U. Beifuss, Angew. Chem. Int. Ed., 2007, 46, 3353. (b) S.-C. Yang, P.-C. Liu and W.-H. Feng, Tetrahedron Lett., 2004, 45, 4951. (c) R. Mukhopadhyay and N. G. Kundu, Synlett, 2001, 1143. (d) V. Nair, R. Dhanya, C. Rajesh, M. M. Bhadbhade and K. Manoj, Org. Lett., 2004, 6, 4743. (e) K. C. Majumdar, K. Ray and S. Ponra, Tetrahedron Lett., 2010, 51, 5437. (f) R. A. Bunce, D. M. Herron and L. Y. Hale, J. Heterocycl. 
Chem., 2003, 40, 1031. (g) V. Krchňák, J. Smith and J. Vágner, Tetrahedron Lett., 2001, 42, 2443. (h) L. Y. Zheng and X. Bai, Chin. Chem. Lett., 2006, 17, 165.

3) For a comprehensive review of the nitro-Mannich reaction see A. Noble and J. C. Anderson, Chem. Rev., 2013, 113, 2887.

4) (a) J. C. Anderson and H. A. Chapman, Org. Biomol. Chem., 2007, 5, 2413. (b) J. C. Anderson, A. Noble and P. Ribelles Torres, Tetrahedron Lett. 2012, 53, 5707.

5) (a) J. C. Anderson, G. J. Stepney, M. R. Mills, L. R. Horsfall, A. J. Blake and W. Lewis, J. Org. Chem., 2011, 76, 1961. (b) J.C. Anderson, A. J. Blake, P. J. Koovits, and G. J. Stepney, J. Org. Chem. 2012, 77, 4711. (c) J. C. Anderson, and P. J. Koovits, Chem. Sci. 2013, 4, 2897. (d) J. C. Anderson and A.S Kalogirou and G. J. Tizzard, Tetrahedron 2014, 17, 9337.

6) (a) J. C. Anderson, L. R. Horsfall, A. S. Kalogirou, M. R. Mills, G. J. Stepney, and G. J. Tizzard, J. Org. Chem., 2012, 77, 6186. (b) J.C. Anderson, A.S. Kalogirou, M.J. Porter and G.J. Tizzard, Beil. J. Org. Chem. 2013, 9, 1737.

7) (a) J. C. Anderson, A. Noble and D. A. Tocher, J. Org. Chem., 2012, 77, 6703. (b) J. C. Anderson, I. B. Campbell, S. Campos, J. Shannon, and D. A. Tocher, Org. Biomol. Chem. 2015, 13, 170. (c) J. C. Anderson, J. P. Barham and C. D. Rundell, Org. Lett. 2015, 17, 4090. (d) J. C. Anderson, J.C. and C. D. Rundell, Synlett 2016, 27, 41.

8) H. Adams, J. C. Anderson, S. Peace and A. M. K. Pennell, J. Org. Chem., 1998, 63, 9932.

9) (a) J. C. Anderson and G. P. Howell, J. Org. Chem. 2005, 70, 549. (b) J. C. Anderson, G. P. Howell, R.M. Lawrence and C. Wilson, J. Org. Chem. 2005, 70, 5665 .

10) The $\mathrm{p} K_{\mathrm{a}}$ could not be determined in water between $\mathrm{pH} 2-12$ indicating that the $\mathrm{p} K_{\mathrm{a}} \mathrm{H}$ is $<2$ (See ESI).

11) D. S. Surry and S. L. Buchwald, Chem. Sci., 2011, 2, 27. 
Table of contents graphic
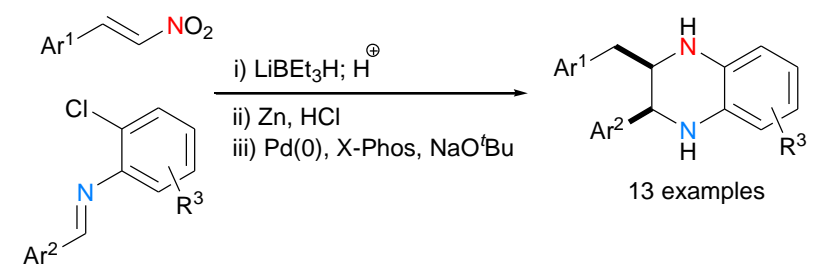

A reductive conjugate addition nitro-Mannich reaction controls diastereoselectivity in a rapid entry to a diverse array of 1,2,3,4-tetrahydroquinoxalines in high yield. 